\title{
Operation time is independent associated with serious postoperative symptom in patients with mandibular third molar removal
}

\author{
Feng Qiao ${ }^{1 \#}$, Lei Li ${ }^{2 \#}$, Jing Zhang $^{1}$, Rui Dong ${ }^{1}$, Jun Sun $^{1}$ \\ ${ }^{1}$ Department of Oral and Maxillofacial Surgery, School and Hospital of Stomatology, Tianjin Medical University, Tianjin, China; ${ }^{2}$ Department of \\ Stomatology, Aerospace Center Hospital, Beijing, China \\ Contributions: (I) Conception and design: F Qiao; (II) Administrative support: None; (III) Provision of study materials or patients: J Zhang, R Dong; \\ (IV) Collection and assembly of data: R Dong, J Sun; (V) Data analysis and interpretation: L Li, J Zhang; (VI) Manuscript writing: All authors; (VII) \\ Final approval of manuscript: All authors. \\ \#These authors contributed equally to this work. \\ Correspondence to: Feng Qiao, MDS. Associate Chief Physician, Department of Oral and Maxillofacial Surgery, School and Hospital of Stomatology, \\ Tianjin Medical University, 12 Qixiangtai Road, Heping District, Tianjin 300070, China. Email: qiaofeng@tmu.edu.cn.
}

Background: To investigate the association between operation time and serious postoperative symptom
severity (PoSS) in patients following the surgical removal of mandibular third molar (M3M), independent of
the degree of impaction.
Methods: A retrospective hospital-based observational study was carried out at the Stomatology Hospital
of Tianjin Medical University. The operation time was recorded and divided by quartiles (Q1-Q4).
The primary outcome was PoSS. Clinical, demographic and radiologic variables were also recorded. A
descriptive bivariate analysis was performed. Associations were analyzed using the adjusted multivariate
logistic regression. The association between time and impaction status was also tested. Potential nonlinear
associations were evaluated with the restricted cubic spline. P values of $<0.05$ were considered as statistically
significant.

Results: A total of 321 patients met our inclusion criteria (114 men and 207 women). The mean operation time was 16.0 minutes (range, 1.1 to 40.5 minutes). The odds ratio (OR) and $95 \%$ confidence interval (CI) were determined after multivariate adjustments for the risk of serious PoSS per 5 -min incremental increase in operation time (OR 1.201, 95\% CI, 1.005-1.434). The multivariable-adjusted ORs (95\% CI) of PoSS across the categories of operation time were as follows: 1.00 (reference), 1.765 (0.770-4.045), 2.567 (1.0476.293 ), and 3.085 (1.180-8.064) ( $\mathrm{P}$ for trend $=0.027)$. The test of interaction effect was not significant (all $\mathrm{P}$ for interaction $>0.05$ ). No evidence of a nonlinear association was observed ( $\mathrm{P}$ for nonlinearity $=0.117$ ).

Conclusions: Operation time was significantly and positively associated with a high risk of serious PoSS independent of impaction status in patients who underwent the surgical removal of M3M.

Keywords: Operation time; postoperative symptom; logistic model; restricted cubic spline; mandibular third molar (M3M)

Submitted Nov 25, 2020. Accepted for publication Feb 03, 2021.

doi: 10.21037/apm-20-2340

View this article at: http://dx.doi.org/10.21037/apm-20-2340 


\section{Introduction}

Extraction of the mandibular third molar (M3M) is a widely performed procedure with several possible postoperative complications (1). These morbidities may aggravate the body's stress response and prolong the recovery (2). The postoperative symptom severity (PoSS) has been proposed and proved to be a reliable, valid, and responsive measurement for assessing health outcomes after the removal of the third molars $(3,4)$. It has been often used to evaluate PoSS after M3M removals $(5,6)$ and has been attracted much attention in recent years. PoSS has been associated with demographic and surgical variables $(7,8)$. Difficulty and complications related to the procedure have been discussed in the literature $(9,10)$.

The classification of impaction status for M3M is mainly based on local anatomy and radiographs (11). Several classification systems have been proposed for evaluating the difficulty index for impacted M3M $(12,13)$. All these classifications illustrate the relationship between radiological anatomy and surgical difficulty. The Pell and Gregory (PG) classification (14) and the Winter classification (15) also consider the main risk factors for postoperative morbidity and complications (16-18). Recent research has shown that the P-G classification is a risk factor for PoSS (8). The ramus relationship/space available to third molars and the degree of bone impaction may be the reason for extended operation time and serious postoperative symptoms. Additionally, operation time has also been shown to be a predictor of postoperative symptoms and is used as a proxy measure for extraction difficulty $(7,16)$. Recent research has shown that the relationship between the M3M position, operation time, and surgical difficulty may be influenced by radiological factors such as the P-G level classification (12). These findings suggest a possible association between operation time and PoSS. However, results from previous studies remain unclarified and relatively sparse. Evidence for the relationship between operation time and PoSS in patients with different P-G classifications is still lacking.

Recently, several regression models have been proposed to evaluate the surgical time of $M 3 M$ extraction $(19,20)$. However, most of these observational studies may have been biased because prognostic factors were unequally distributed between patients who were exposed or not exposed to an intervention. Although the effectiveness and clinical role of time have been partly discussed, it would be preferable that adjusted or stratified analysis was used to create prognostically homogeneous groups and to combine the effect estimates across the groups. Moreover, it would be much more informative to measure the non-linear relationship between time and postoperative symptoms.

Therefore, we hypothesized that the operation time may be independently associated with PoSS among different impaction status classifications after adjustment and stratification for confounders. The aim of our study was to examine the association between operation time and PoSS at different degrees of impacted in patients with M3M removal.

We present the following article in accordance with the STROBE reporting checklist (available at http://dx.doi. org/10.21037/apm-20-2340).

\section{Methods}

\section{Study design}

In accordance with the research purpose, a hospital-based retrospective observational study was carried out using a self-reported questionnaire in Tianjin, China. Adults who had surgical extractions of M3Ms between May 2016 to June 2017 at the Department of Oral and Maxillofacial Surgery were eligible. Patient recruitment complied with the following inclusion criteria: indication for M3Ms surgery under local anesthesia and good health without systemic diseases. Patients with systemic diseases or behavior disorders, the absence of the mandibular second molar, and who were unable to respond to questionnaires, were excluded. The study was conducted in accordance with the Declaration of Helsinki (as revised in 2013). The study was approved by institutional committee (No.: TMUhMEC2018110). Informed consent was taken from all the patients.

\section{Study variables}

The primary independent variable was surgery operation time. We defined the operation time as the duration from the start of the incision to the end of the wound suture. It was further categorized into equal quartiles (Q1-Q4). The reference group was defined as $<8.43$ minute.

\section{Response variable}

The primary outcome measure for the study was the total PoSS. The PoSS included seven subscales for evaluating the oral health-related quality of life (HRQOL) after 
M3M removal. The definition of PoSS was assessed using the postoperative symptom severity scale (PoSSe) questionnaire (3). The PoSSe score was calculated from the self-reported questionnaire items as follows:

Full PoSSe score $=$ eating scores + speech scores + sensation scores + appearance scores + pain scores + sickness scores + interference scores.

The PoSSe score was recorded when the sutures were removed 7 days after surgery. We divided patients into the high-risk and low-risk groups according to the median value of their full PoSSe scores. Patients with full PoSSe scores $\geq$ median were classified into the high-risk group, which was assigned a value of 1 ; while patients with full PoSSe scores $<$ median were classified into the lowrisk group, which was assigned a value of 0 . Higher total PoSSe scores represent serious PoSS (3). Our sample size calculation was determined by our primary objective (binary outcome), based on 10 outcome events per variable (EPV) investigations (21).

\section{Control of confounders}

Data were collected using a standardized form for each operation. The designated recorder was present at each operation. The covariates used in this study were classified as follows: (I) demographic data; (II) anatomical and radiographic variables that have been reported in previous literature; and (III) surgical operation-related factors.

Panoramic radiographs were used to classify the $M 3 M$ according to the P-G classification system $(14,22)$. This system classifies the teeth based on the amount of tooth covered by the anterior border of the ramus (Class I-III) and the impaction depth, relative to the adjacent tooth (Positions A, B, or C).

Anthropometric parameters were recorded using a standard protocol. The following confounders were recorded: patient variables [sex, age, body mass index (BMI), mouth opening, smoking status, and pre-surgery symptoms] and tooth-related variables (the Winter classification, number of roots, root curvature, and root morphology). The degree of mouth opening and tooth-related variables have been described in previous research $(7,8,19)$. Patients were asked whether they had any history, symptoms, or signs of pericoronitis. Pre-surgery panoramic radiographs were used to evaluate tooth-related variables and $\mathrm{P}-\mathrm{G}$ and Winter classifications. Furthermore, the root curvature was classified into two categories in accordance with the measured angle between the tooth length axis and the tangent of the curved root. Root morphology was defined as conical, spherical, and bifurcation, as described by Qiao et al. (7). To address potential sources of bias, Kappa values were used to assess consistency (Kappa $=0.72, \mathrm{P}<0.05$ ), which indicating high consistency.

According to the standard operating procedures, local anesthesia was administered under the same conditions using either $2 \%$ lidocaine and $4 \%$ articaine. A contraangle high-speed turbine (NSK Ltd., Tokyo, Japan) and a tungsten steel crack needle drill (NSK Ltd.) were used for conventional rotary osteotomy. The flap design was categorized as no flap, relaxing incision, triangular flap. The surgical procedure was recorded as elevator/forceps alone, bone removal or tooth sectioning, bone removal and tooth sectioning according to the degree of impaction of the M3M. Surgical experience was defined as the number of years since the completion of residency, which was categorized as $0-5,5-10$ or $>10$ years. We completed all protocols under the same environments to address potential sources of bias. Patients were given the usual postoperative instructions immediately after the surgery. Antibiotics and anti-inflammatory drugs were prescribed (usually cefuroxime $250 \mathrm{mg} 2$ times daily for 3 days and ibuprofen $60 \mathrm{mg}$ three times daily for 3 days).

\section{Data management and statistical analysis}

Data were collected using a standardized form for each operation. All data were entered into EpiData, version 3.1 (The EpiData Association, Odense, Denmark), and then transferred to SPSS (Version 25.0), and R, version 4.0.1 (R Foundation for Statistical Computing, Vienna, Austria) for statistical analyses. Distributions of continuous variables were assessed for normality using the ShapiroWilk $(n \leq 2,000)$ tests. The distributions of all continuous variables were not normal in our study. Categorical variables were presented as percentages and continuous variables as medians (25\%, $75 \%$ quantiles). The differences among categories regarding PoSS were examined using the Kruskal-Wallis (comparison of $>2$ groups) for continuous and ordinally distributed variables and the Chi-squared test was used for categorical variables. For further analysis, the presence of severe symptoms was used as a response variable and the operation time was used as independent variable. Logistic regression analysis was used to examine the association between the duration of surgery and the presence of serious symptoms after adjustment for potentially confounding factors. Interactions between the 


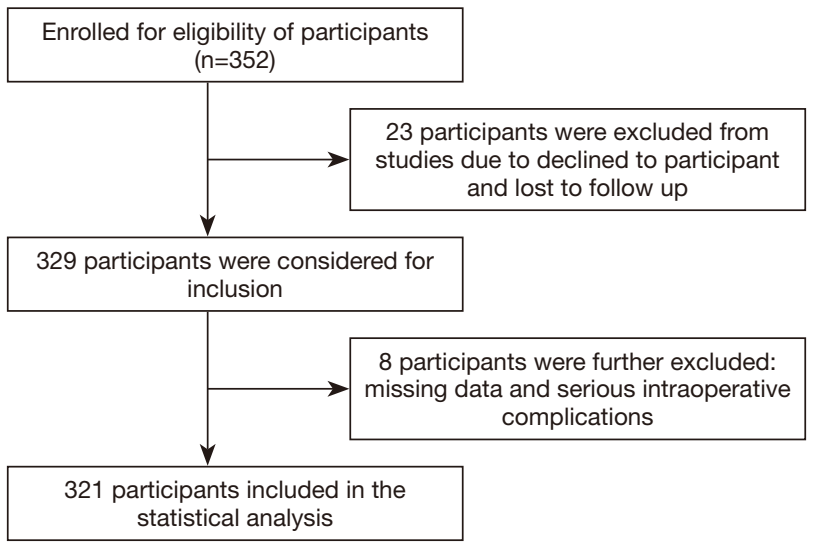

Figure 1 Study participants' selection flowchart in observational final analysis.

duration of operation and potential confounders were tested by the addition of cross-product terms in the regression model. We also used restricted cubic splines with three knots to flexibly model the association of operation time with PoSS. Adjusted odds ratios (ORs) with 95\% confidence intervals (CIs) were also constructed. Nominal, two-tailed $\mathrm{P}$ values were used. $\mathrm{P}$ values less than 0.05 were considered statistically significant.

\section{Results}

In our study, there were a total of 321 patients who met the inclusion criteria and were eligible to enroll (Figure 1). The mean (SD) operation time for these patients was 16.1 (9.4) minutes. Of the participants, $35.5 \%$ (114 of 321) were males, in whom the mean (SD) operation time was 16.4 (8.5) minutes; and $64.5 \%$ (207 of 321) were females, in whom the mean (SD) operation time was 15.9 (9.8) minutes (Table 1). The overall presence of serious postoperative symptoms was $48.3 \%$ (155 of 321 ). The PoSS in the quartile categories of operation time $(\leq 8.33 ; 8.34-14.83$; $14.84-22.12$; $\geq 22.13$ minutes) was $27.7 \%, 36.7 \%, 44.3 \%$, $33.8 \%$ in men and $72.3 \%, 63.3 \%, 55.7 \%, 66.3 \%$ in females, respectively. Baseline characteristics of the study population for total samples and subgroups according to the categories of operative time are shown in Table 1.

The multivariable-adjusted ORs (95\% CI) of PoSS for per- 5 min incremental in operation time were as follows: 1.241 (1.098-1.403) in the crude model, 1.219 (1.070-1.388) when adjusted for impaction as determined by the P-G classification, and $1.201(1.005-1.434)$ when additionally adjusted for other confounders (Table 2).
In the crude model, when comparing the operation time's highest quartile to the lowest quartile, time was significantly associated with serious PoSS (OR 3.278; 95\% CI, 1.724-6.235; P for trend <0.001) (Table 2). In analyses adjusted for impaction status (measured by the P-G classification), the adjusted OR for serious PoSS was 3.031 (95\% CI, 1.551-5.923) in individuals with operation time in the top quartiles compared to those in the bottom quartiles ( $\mathrm{P}$ for trend $=0.001)$ (Table 2). After additional adjustments for demographic, radiographic, and operative variables, the operation time remained significantly associated with serious PoSS (OR 3.085; 95\% CI, 1.180-8.064; P for trend $=0.027$ ) (Table 2).

When we analyzed operation time in association with PoSS across subgroups of risk factors, we detected no significant subgroup interactions. The interactions between the P-G ramus classification, the Winter classification, flap design, surgical procedure, experience, and operation time in the models were not statistically significant (all $\mathrm{P}$ for interaction $>0.1$ ) (Figure 2).

Analyses using the restricted cubic spline suggested a dose-response association between operation time and the PoSS OR (Figure 3). The risk of PoSS started to increase ( $\mathrm{P}$ for non-linearity $=0.117$ ). The predicted OR of PoSS per 5 min incremental increase was 1.201 (95\% CI, 1.181.26). Regarding the non-linear relationship between the operation time and PoSS, the plot showed a substantial increase in the risk within the surgical duration range.

\section{Discussion}

Our analyses found that operation time was significantly 
Table 1 Baseline characteristics of the study population for total samples and subgroups according to categories of operation time (Q1-Q4)*

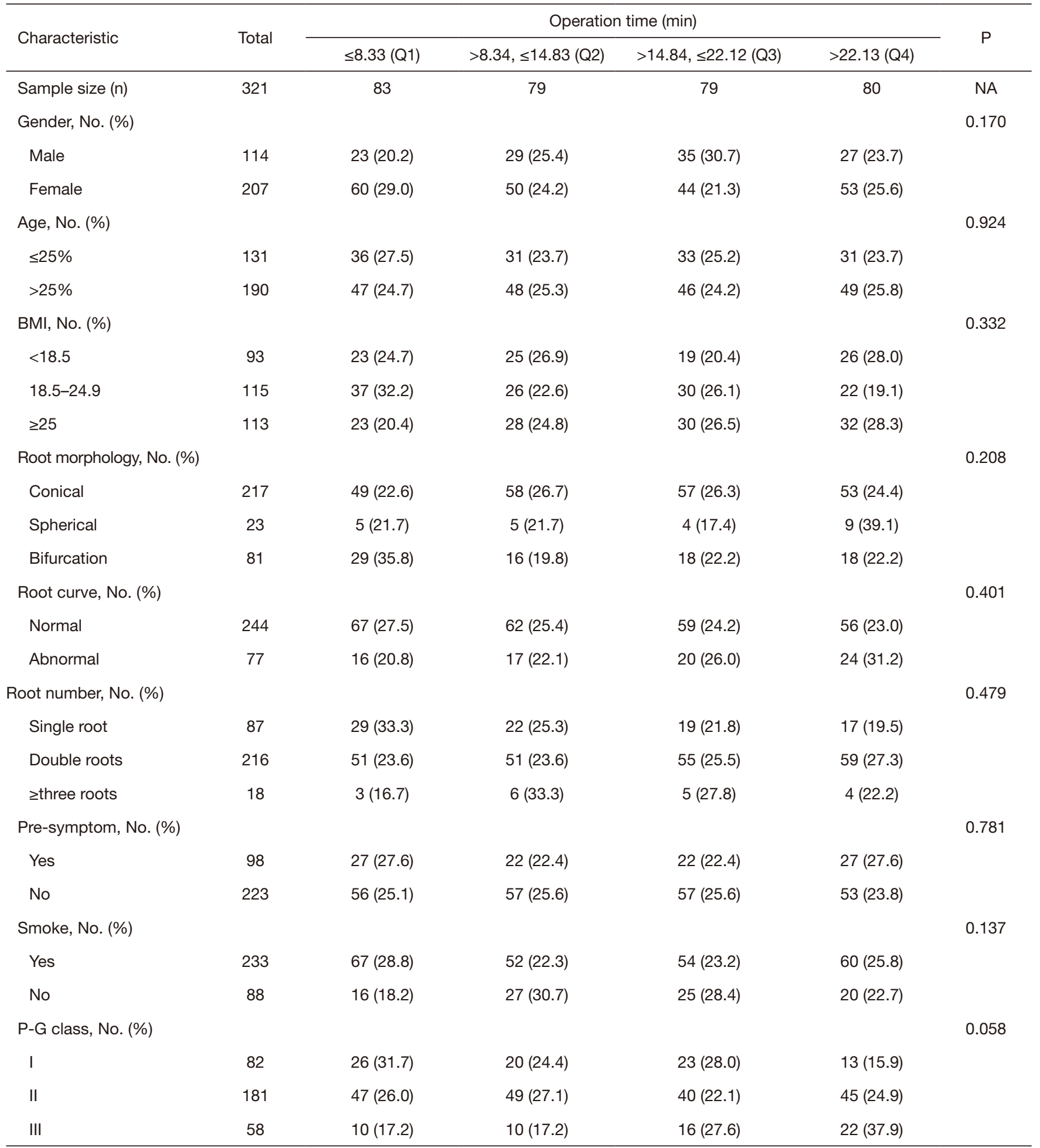

Table 1 (continued) 
Table 1 (continued)

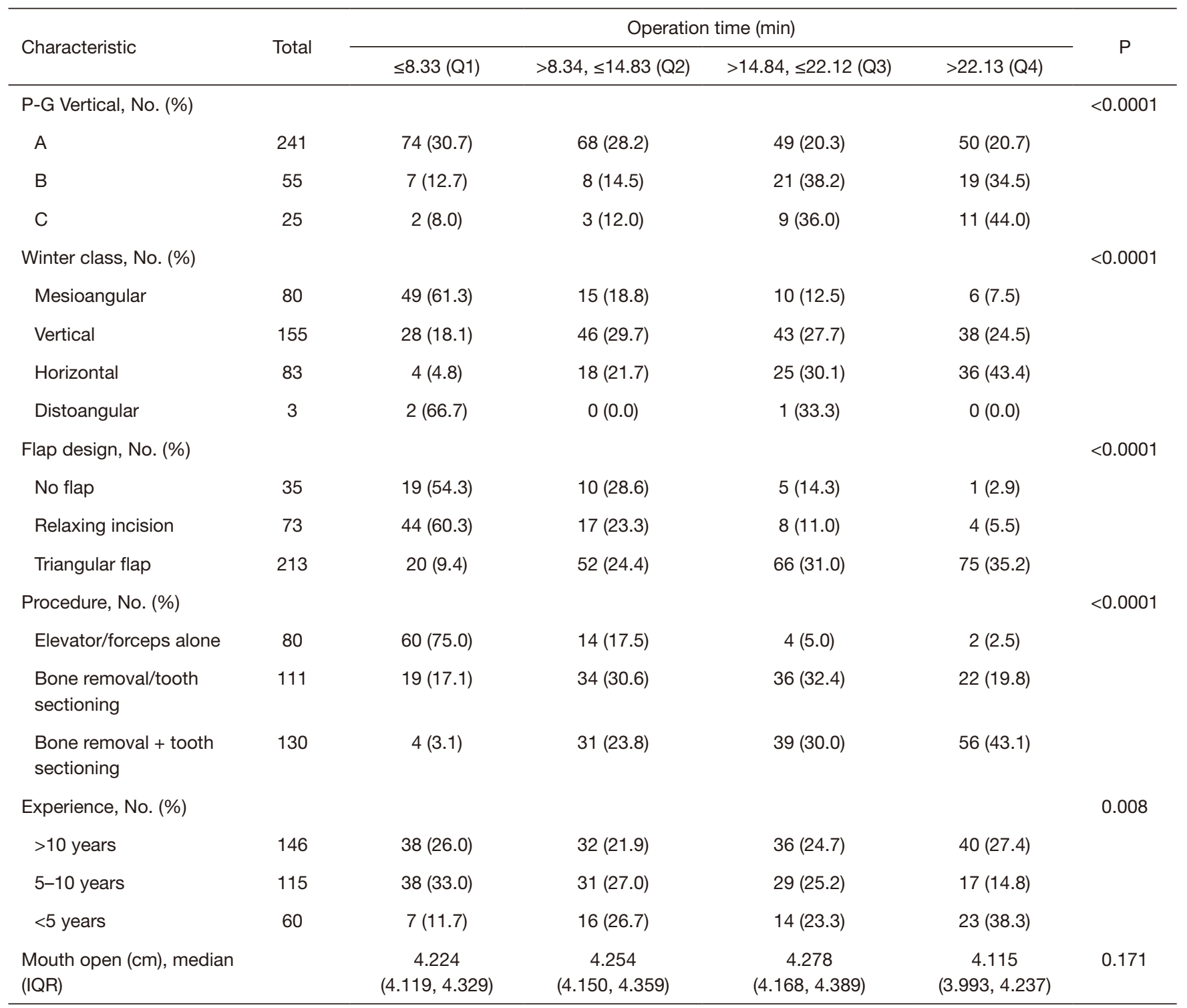

*, continuous variables are presented as median (IQR). Kruskal-Wallis test were used to compare continuous variables across operation time quartiles. Fisher chi-square test was used to test the association of categorical variables with operation time quartiles. NA, not available; median (IQR), medians (interquartile range) (25-75\% quantiles); BMI, body mass index; root morph, morphology of root; root curve, curvature of root; root number, number of root; pre-symptom, preoperative symptoms of mandibular third molar; P-G class, Pell and Gregory classification; Winter class, Winter classification; mouth open, the opening of mouth.

and positively associated with serious PoSS, independent of several risk factors, even adjustment for the P-G ramus classification. These results help to clarify the relationship between the operation time and PoSS.

Some studies have shown that prolonged operation time increases the likelihood of complications, and possibly results in postoperative symptoms (23). Several studies have also reported an association between impact status and postoperative complications (24-26). Current evidence support that operation time is strongly associated with the difficulty of the surgery (27). Phillips et al. showed that an increase in the risk of prolonged recovery for HRQOL outcomes and delayed clinical outcomes after third molar surgery $(28,29)$. In general, insufficient space 
Table 2 Adjusted odds ratios ( $95 \%$ confidence interval) for the categories of the operation time with postoperative symptom ${ }^{\mathrm{a}}$

\begin{tabular}{|c|c|c|c|c|}
\hline Operation time (min) & $\mathrm{N}$ & Model $1^{\mathrm{d}}$, OR $(95 \% \mathrm{Cl})$ & Model $2^{\mathrm{e}}$, OR $(95 \% \mathrm{Cl})$ & Model $3^{\dagger}$, OR $(95 \% \mathrm{Cl})$ \\
\hline \multicolumn{5}{|l|}{ Time (quartile) } \\
\hline$\leq 8.33(\mathrm{Q} 1)$ & 83 & 1.00 (Reference) & 1.00 (Reference) & 1.00 (Reference) \\
\hline 8.34-14.83 (Q2) & 79 & $1.827(0.966-3.456)^{\mathrm{c}}$ & $1.803(0.947-3.434)$ & $1.765(0.770-4.045)$ \\
\hline$\geq 22.13$ (Q4) & 80 & $3.278(1.724-6.235)$ & 3.031 (1.551-5.923) & 3.085 (1.180-8.064) \\
\hline$P$ for trend ${ }^{b}$ & & $<0.001$ & 0.001 & 0.027 \\
\hline
\end{tabular}

${ }^{a}$, multivariate logistic regression analysis was used to sequentially adjusted for covariates; ${ }^{b}, \mathrm{P}$ for trend, $\mathrm{P}$ for linear trend was calculated by modeling the median of the operative time for each quartiles as a continuous variable; ${ }^{c}$, continues variables; ${ }^{\mathrm{d}}$, crude model; ${ }^{\mathrm{e}}$, adjusted for impaction status (Pell-Gregory's classification). Adjusted odds ratios (95\% confidence interval) (all such values); ${ }^{\dagger}$, additionally adjusted for gender, age, BMI (body mass index), Winter classification, number of root, curvature of root, and morphology of root, preoperative symptoms of mandibular third molar, smoking status, flap design, surgery procedure, experience, mouth opening. OR, odds ratio; Cl, confidence interval.

Characteristics
Gender
Male
Female
Interaction
Smoke,No(\%)
Yes
No
Interaction
Pell-Gregory ramus classification,No(\%)
I
II
III
Interaction
Winter classification,No(\%)
Mesioangular
Vertical
Horizontal
Interaction
Flap design,No(\%)
No flap
Relaxing incision
Triangular flap
Interaction
Surgical procedure,No(\%)
Elevator/forceps alone
Bone removal/tooth sectioning
Bone removal+tooth sectioning
Interaction
Experience,No(\%)
$>10$ years
$5 ~ 10 y e a r s$
$<5 y e a r s$
Interaction

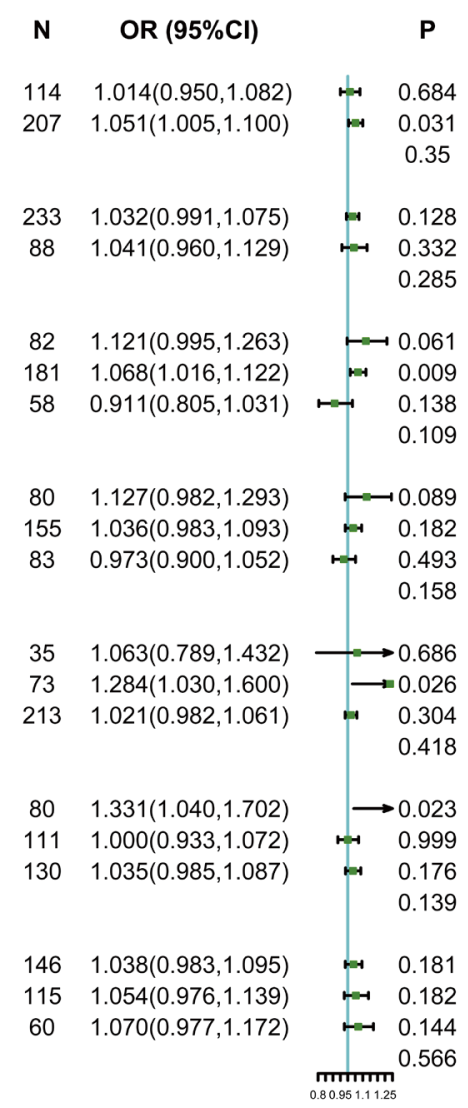

Figure 2 Forest plot of interaction stratified by subgroup analysis baseline characteristics for association between operation time and severity postoperative symptom (PoSS). OR, odds ratio; CI, confidence interval. 


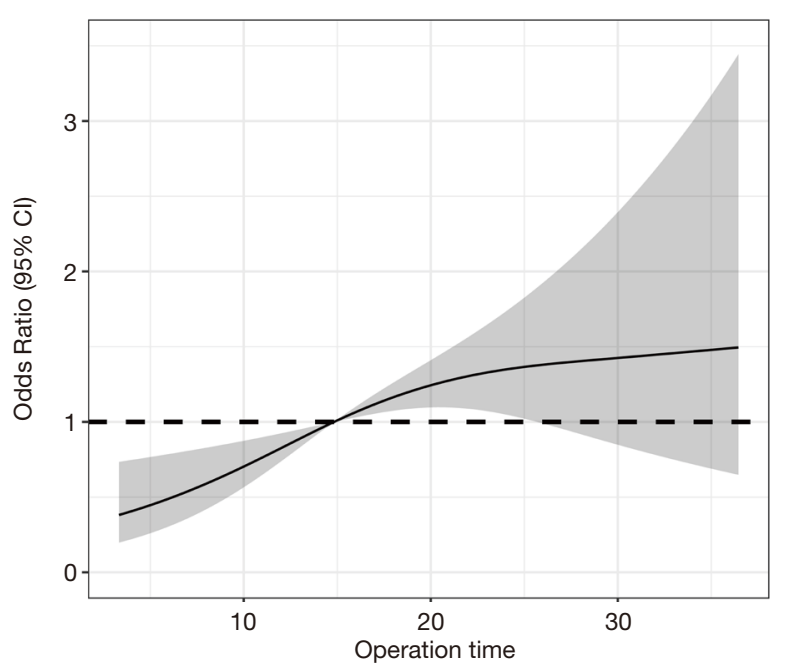

Figure 3 Restricted cubic spline analysis of the association between operation time and PoSS. The middle solid line indicates the point estimates of odds ratios and the shaded part indicate the lower and upper limits of the corresponding $95 \%$ confidence intervals. The horizontal dashed line is at odds ratio $=1$. Three knots were used for the restricted cubic spline analysis. The non-linear association was not observed ( $\mathrm{P}$ for non-linearity $=0.117$ ). CI, confidence interval.

available in relation to the ramus means greater trauma and complications during the surgery. Since studies have shown that the severity of postoperative symptoms is related to the P-G classification (8), we first adjusted for this confounder. Our results are consistent with those of a previous study (29).

It is well known that many factors contribute to postoperative symptoms and prolonged recovery (28). We subsequently additionally adjusted for sex, age, BMI, the Winter classification, number of roots, root curvature, root morphology, pre-symptom, smoking status, flap design, operation procedure, experience, and mouth opening. The results of our study show that comparing the top and bottom quartiles, the risk PoSS increased by 2.031 times when adjusted for the P-G classification (OR 3.031, 95\% CI, 1.551-5.923). After adjusting for the confounding factors (such as the P-G classification), the independent effect of operation time on the severity of PoSS was quantified. This result indicates that operation time may independently increase the risk of PoSS. After a similar multivariable adjustment for demographic and surgical characteristics, the effect of operation time on the risk of PoSS in patients with M3M extraction was weakened. This indicates that multi-factor adjustments played a role in controlling the confounding factors. Our study implied that longer surgical times may dictate a more aggressive pain regimen, including the use of opioids.

Interaction analyses was revealed evidence of the association between time and PoSS. Subgroup analysis of the multivariable-adjusted model shows that the relationship between time and PoSS is reasonably credible in different subgroups, indicating that the result is stable and reliable (Figure 2).

In addition, we used the restricted cubic splines to flexibly model and visualize the relationship between operation time and PoSS. The restricted cubic spline analysis did not indicate a non-linear association between operation time and PoSS ( $\mathrm{P}$ for nonlinear trend $=0.117$ ). This demonstrates that the longer the operation time, the more serious the postoperative symptoms. Thus, minimizing the operation time is also a possible strategy for reducing postoperative symptoms. In terms of surgical difficult, several classification systems have been proposed. Further studies based on these classification systems will further confirm the relationship between operation time and PoSS.

\section{Limitations}

There are some limitations to the interpretation of the results. First, due to the limitations of a retrospective study, possible selection bias and the single-center sample population, may have been present in this research. Second, self-reported questionnaires are subjective indicators, which may be more prone to information bias and/ or recall bias. Replication of these studies is required to enhance the generalizability and external validity of study findings. Third, although we adjusted for several potential confounding factors where appropriate, residual confounding from other unmeasured factors may remain.

\section{Conclusions}

In conclusion, the operation time is independently associated with PoSS in patients who underwent M3M removal. Minimizing the duration of surgery would not only improve the postoperative recovery but also maximize patient satisfaction.

\section{Acknowledgments}

We would like to thank Editage (www.editage.cn) for 
English language editing.

Funding: None.

\section{Footnote}

Reporting Checklist: The authors have completed the STROBE reporting checklist. Available at http://dx.doi. org/10.21037/apm-20-2340

Data Sharing Statement: Available at http://dx.doi. org/10.21037/apm-20-2340

Conflicts of Interest: All authors have completed the ICMJE uniform disclosure form (available at http://dx.doi. org/10.21037/apm-20-2340). The authors have no conflicts of interest to declare.

Ethical Statement: The authors are accountable for all aspects of the work in ensuring that questions related to the accuracy or integrity of any part of the work are appropriately investigated and resolved. The study was conducted in accordance with the Declaration of Helsinki (as revised in 2013). The study was approved by institutional ethics board of School and Hospital of Stomatology Tianjin medical university (No.: TMUhMEC2018110) and informed consent was taken from all the patients.

Open Access Statement: This is an Open Access article distributed in accordance with the Creative Commons Attribution-NonCommercial-NoDerivs 4.0 International License (CC BY-NC-ND 4.0), which permits the noncommercial replication and distribution of the article with the strict proviso that no changes or edits are made and the original work is properly cited (including links to both the formal publication through the relevant DOI and the license). See: https://creativecommons.org/licenses/by-nc-nd/4.0/.

\section{References}

1. Stacchi C, Daugela P, Berton F, et al. A classification for assessing surgical difficulty in the extraction of mandibular impacted third molars: Description and clinical validation. Quintessence Int 2018;49:745-53.

2. Bui CH, Seldin EB, Dodson TB. Types, frequencies, and risk factors for complications after third molar extraction. J Oral Maxillofac Surg 2003;61:1379-89.

3. Ruta D, Bissias E, Ogston S, et al. Assessing health outcomes after extraction of third molars: the postoperative symptom severity (PoSSe) scale. Br J Oral Maxillofac Surg 2000;38:480-7.

4. Grossi GB, Maiorana C, Garramone RA, et al. Assessing postoperative discomfort after third molar surgery: a prospective study. J Oral Maxillofac Surg 2007;65:901-17.

5. Hassan B, Al-Khanati NM, Bahhah H. Effect of lingual-based flap design on postoperative pain of impacted mandibular third molar surgery: Split-mouth randomized clinical trial. Med Oral Patol Oral Cir Bucal 2020;25:e660-7.

6. Brucoli M, De Andreis M, Bonaso M, et al. Comparative assessment of dexamethasone administration routes for the management of postoperative symptoms following third molar surgery. J Stomatol Oral Maxillofac Surg 2019;120:529-33.

7. Qiao F, Huang X, Li B, et al. A Validated Model to Predict Postoperative Symptom Severity After Mandibular Third Molar Removal. J Oral Maxillofac Surg 2020;78:893-901.

8. Qiao F, Fu QB, Guo YX, et al. Risk factors for the deterioration of oral health-related quality of life after mandibular third molar removal. Int J Clin Exp Med 2017;10:3281-8.

9. Blondeau F, Daniel NG. Extraction of impacted mandibular third molars: postoperative complications and their risk factors. J Can Dent Assoc 2007;73:325.

10. Cho H, Lynham A, Hsu E. Postoperative interventions to reduce inflammatory complications after third molar surgery: review of the current evidence. Aust Dent J 2017;62:412-9.

11. Hattab FN, Alhaija ESA. Radiographic evaluation of mandibular third molar eruption space. Oral Surg Oral Med Oral Pathol Oral Radiol Endod 1999;88:285-91.

12. Ku JK, Chang NH, Jeong YK, et al. Development and validation of a difficulty index for mandibular third molars with extraction time. J Korean Assoc Oral Maxillofac Surg 2020;46:328-34.

13. Kim JY, Yong HS, Park KH, et al. Modified difficult index adding extremely difficult for fully impacted mandibular third molar extraction. J Korean Assoc Oral Maxillofac Surg 2019;45:309-15.

14. Pell GJ. Impacted mandibular third molars: classification and modified techniques for removal. Dent Digest 1933;39:330-8.

15. Winter G. Principles of exodontia as applied to the impacted mandibular third molar. American Medical Book Co., St Louis; 1926:241-79.

16. Sanchez-Torres A, Soler-Capdevila J, Ustrell-Barral M, et al. Patient, radiological, and operative factors associated 
with surgical difficulty in the extraction of third molars: a systematic review. Int J Oral Maxillofac Surg 2020;49:655-65.

17. Benediktsdottir IS, Wenzel A, Petersen JK, et al. Mandibular third molar removal: risk indicators for extended operation time, postoperative pain, and complications. Oral Surg Oral Med Oral Pathol Oral Radiol Endod 2004;97:438-46.

18. Yuasa H, Kawai T, Sugiura M. Classification of surgical difficulty in extracting impacted third molars. Br J Oral Maxillofac Surg 2002;40:26-31.

19. Susarla SM, Dodson TB. Predicting third molar surgery operative time: a validated model. J Oral Maxillofac Surg 2013;71:5-13.

20. Laskin DM, Abubaker AO, Strauss RA. Accuracy of predicting the duration of a surgical operation. J Oral Maxillofac Surg 2013;71:446-7.

21. Peduzzi P, Concato J, Kemper E, et al. A simulation study of the number of events per variable in logistic regression analysis. J Clin Epidemiol 1996;49:1373-9.

22. Soos B, Janovics K, Toth A, et al. Association Between Third Molar Impaction Status and Angle or Condylar Fractures of the Mandible: A Retrospective Analysis. J Oral Maxillofac Surg 2020;78:1162.e1-1162.e8.

23. Bello SA, Adeyemo WL, Bamgbose BO, et al. Effect of

Cite this article as: Qiao F, Li L, Zhang J, Dong R, Sun J. Operation time is independent associated with serious postoperative symptom in patients with mandibular third molar removal. Ann Palliat Med 2021;10(4):4080-4089. doi: 10.21037/ apm-20-2340 age, impaction types and operative time on inflammatory tissue reactions following lower third molar surgery. Head Face Med 2011;7:8.

24. Akadiri OA, Obiechina AE. Assessment of difficulty in third molar surgery--a systematic review. J Oral Maxillofac Surg 2009;67:771-4.

25. de Carvalho RW, de Araujo Filho RC, do Egito Vasconcelos BC. Assessment of factors associated with surgical difficulty during removal of impacted maxillary third molars. J Oral Maxillofac Surg 2013;71:839-45.

26. Carvalho RWF, do Egito Vasconcelos BC. Assessment of factors associated with surgical difficulty during removal of impacted lower third molars. J Oral Maxillofac Surg 2011;69:2714-21.

27. Alvira-González J, Figueiredo R, Valmaseda-Castellón E, et al. Predictive factors of difficulty in lower third molar extraction: A prospective cohort study. Med Oral Patol Oral Cir Bucal 2017;22:e108.

28. Phillips C, Gelesko S, Proffit WR, et al. Recovery after third-molar surgery: the effects of age and sex. Am J Orthod Dentofacial Orthop 2010;138:700.e1-e8.

29. Phillips C, White RP Jr, Shugars DA, et al. Risk factors associated with prolonged recovery and delayed healing after third molar surgery. J Oral Maxillofac Surg $2003 ; 61: 1436-48$. 